\title{
INTERNATIONAL AND DOMESTIC TRADING AND WEALTH DISTRIBUTION *
}

\author{
B. DÜRING $\dagger$ AND G. TOSCANI $\ddagger$
}

\begin{abstract}
We introduce and discuss a kinetic model for wealth distribution in a simple market economy which is built of a number of countries or social groups. Our approach is based on the model with risky investments introduced by Cordier, Pareschi and one of the authors in [13] and borrows ideas from the kinetic theory of mixtures of rarefied gases. Wealth is exchanged by individuals inside these countries (domestic trade) as well as in between different countries (international trade). Under a suitable scaling we derive a system of Fokker-Planck type equations and discuss its extension to a two-dimensional model with distributed trading propensity. Theoretical and numerical results for two groups show that the wealth distribution develops a bimodal (and in general, a polymodal) shape.
\end{abstract}

Key words. Wealth and income distributions, Boltzmann equation, mixture.

MSC 2000 subject classifications. 91B60, 82C40, 35B40.

\section{Introduction}

In recent years, a number of models have been proposed to account for the evolution of the distribution of wealth in a simple market economy. One class that might be considered to constitute a mesoscopic approach is based on generalized Lotka-Volterra models [24, 31]. A second more popular approach relies on methods borrowed from statistical mechanics for particle systems [23, 14, 8, 7, 22, 29, 11, 13]. The founding idea behind this last approach is that a trading market composed by a sufficiently large number of agents can be described using the laws of statistical mechanics as it happens in a physical system composed of many interacting particles. If one agrees with the claim that there are deep analogies between economics and physics, then various well established physical methods can be applied to analyze wealth distributions in economies. In particular, by identifying wealth in a closed economy with energy, the application of statistical physics methods leads to a better understanding of the development of tails in wealth distributions of real economies. In kinetic models of simple market economies, in fact, the knowledge of the large-wealth behavior of the steady state density is of primary importance, since it determines a posteriori if the model fits data of real economies. By identifying wealth with energy it becomes clear that the problem of describing the large-time behavior of the wealth distribution in a kinetic model of the type considered in $[23,14,8,7,22,29,11,13]$ is the analogue of the problem of describing the large-time behavior of the density in the spatially homogeneous Boltzmann equation. In particular, for nonconservative kinetic models, this analogy has been recently enlightened in $[29,27]$, while convergence to steady wealth distributions in conservative models has been dealt with in $[25,26,16]$.

The features typically incorporated in kinetic trade models are saving effects and randomness. Saving means that agents never exchange their entire wealth in a trade, but are guaranteed to retain at least a certain minimal fraction of their wealth at the end of each trade. This concept has been introduced in [8], where a fixed saving rate for all agents has been proposed, and generalized in [9] by introducing an individual

\footnotetext{
* Received date / Revised version date

${ }^{\dagger}$ Institut für Analysis und Scientific Computing, Technische Universität Wien, 1040 Wien, Austria, email: bduering@anum.tuwien.ac.at

¥Dipartimento di Matematica, Università degli studi di Pavia, 27100 Pavia, Italy, email: giuseppe.toscani@unipv.it
} 
saving rate. Randomness means that the amount of wealth changing hands is nondeterministic. Among others, this idea has been developed in [13], to include the effects of a risky market. Numerous numerical simulations for models of the prescribed type have been carried out with different mechanism for saving and varying degree of randomness (see the recent book [10] for an overview of the recent results). In most of these models, however, there is conservation of wealth in individual trades.

The distributed saving gives rise to an additional interesting feature when a special case is considered where the saving parameter is assumed to take only two fixed values, preferably widely separated. In this case, the steady distribution of wealth can result in a bimodal distribution [21]. The numerical output evolves towards a robust and distinct two-peak distribution as the difference in the two saving parameters is increased systematically. A population can be imagined to have two distinctly different kinds of people: some of them tend to save a very large (fixed) fraction of their wealth and the others tend to save a relatively small (fixed) fraction of their wealth. Bimodal distributions (and a polymodal distribution, in general) are, in fact, reported with real data for the income distributions in Argentina [20].

Bimodal distributions have been recently found in a different context, by studying a suitable modification of the nonconservative trade model of Slanina [29] to account for different outcomes which depend on the individual wealth [12]. Numerical experiments of the model then show that the (normalized) wealth distribution tends to develop a bimodal distribution with a power law profile for large wealths. Despite the fact that the model considered in [24] is nonconservative, while the analysis of Gupta [21] refers to a conservative one, there is a common feature which produces the bimodal effect, which can be easily recognized in a separation of trading rules between poor and rich people.

In this paper, we will study the problem of formation of bimodal distributions in a society, by means of the trade model introduced in [13], suitably modified to describe different groups of agents. This model, in fact, is sufficiently flexible to take into account both the randomness of the market and a (variable) saving rate. In addition, it allows for a suitable asymptotic analysis (the continuous trading limit), from which one can pass from the kinetic description in terms of a Boltzmann-type equation to a simpler description in terms of Fokker-Planck type equations. These last equations possess in general steady states which can be explicitly evaluated.

The paper is organized as follows. In the next section we introduce the model which leads to a system of Boltzmann equations. This model is the analogous of a mixture of ideal gases of Maxwell type recently studied in [5]. The continuous trading limit, and the corresponding associated system of Fokker-Plank type equations is studied in Section 3. Section 4 deals with a generalization of the Fokker-Planck system in the case of a distributed saving rate. Numerical examples will be presented in Section 5.

\section{Kinetic models for the evolution of wealth}

The goal of a kinetic model of a simple market economy is to describe the evolution of the distribution of wealth by means of microscopic interactions among agents or individuals which exchange wealth. Each trade is in this picture interpreted as an interaction where a fraction of the wealth changes hands. One generally assumes that this wealth after the interaction is nonnegative, which corresponds to impose that no debts are allowed. In consequence of the trade rules, one expects to obtain for large time a stationary wealth distribution $f_{\infty}(v)$ (denoting the density of agents 
with wealth $v>0$ ) with Pareto tails,

$$
F_{\infty}(w)=\int_{w}^{\infty} f_{\infty}(v) d v \propto w^{-\alpha}
$$

The exponent $\alpha$ is referred to as Pareto index, named after the economist Vilfredo Pareto [28], who proposed formula (2.1) more than a hundred years ago. According to recent empirical data, the wealth distribution among the population in a western country follows in fact a Pareto law, with an index $\alpha$ ranging between 1.5 and 2.5. As briefly outlined in the introduction, a number of different trade models have been proposed to describe economic interactions between agents. The first by Chakrabarti and colleagues $[8,7]$ conserves wealth during the exchange and allows savings that can be a fixed and equal percentage of the initial wealth held by each agent. However, this trade yields the exponentially decaying Gibbs distribution. Allowing the saving percentage to take on a random character [9] then introduces a power law character to the distribution for high incomes, that can be shown to be exactly one. The characteristic of this model have been extensively studied in [26], where it has been clearly established that the appearance of Pareto tails is unnatural and fragile in this framework. Only the presence of random terms in the trade, which destroy the pointwise conservation of wealth, was subsequently shown to be responsible of a robust convergence to a steady distribution with tails [25].

A slightly different phenomenon which leads to formation of tails has been remarked by Slanina [29]. This model assumes a different exchange rule, that allows creation of money during each exchange process and the solution is not stationary. Consequently, one must normalize the amount of money held by an agent with the mean value of money within the system at any time. In this way a stationary solution with tails for the distribution of the normalized money can be obtained. A similar phenomenon appears in kinetic theory of dissipative gases, where the formation of tails in Maxwell models is known as Ernst-Brito conjecture [18, 19, 3, 4, 2]. An exhaustive study of formation of tails in general nonconservative one-dimensional kinetic equations can be found in [27]. It can be argued that the analysis of [27] could be extended to more complicated models, like the one proposed by [12], to recover a qualitative analysis. We will go back to this issue in a forthcoming paper.

2.1. The Cordier-Pareschi-Toscani model The study of the time-evolution of the wealth distribution among individuals in a simple economy, together with a reasonable explanation of the formation of tails in this distribution has been recently achieved by means of kinetic collision-like models in [13]. The Cordier-PareschiToscani model (CPT model) is based on a binary trade which is a balance between both saving and random effects. When two agents with pre-trade wealths $v$ and $w$ interact, then their post-trade wealths $v^{*}$ and $w^{*}$ are given by

$$
\begin{aligned}
v^{*} & =(1-\gamma) v+\gamma w+\eta_{1} v, \\
w^{*} & =(1-\gamma) w+\gamma v+\eta_{2} w .
\end{aligned}
$$

Herein, $\gamma \in(0,1)$ is the constant transaction rate parameter. In other words, the constant saving propensity equals $1-\gamma$. The quantities $\eta_{1}$ and $\eta_{2}$ are random variables with mean zero and variance $\sigma^{2}$. They model risky investments that each agent performs. In addition, to guarantee that the post-trade wealths are non-negative, it is assumed that $\eta_{i} \geq-1+\gamma(i=1,2)$. 
A crucial feature of the CPT model is that it only preserves the total wealth in the statistical mean,

$$
\left\langle v^{*}+w^{*}\right\rangle=\left(1+\left\langle\eta_{1}\right\rangle\right) v+\left(1+\left\langle\eta_{1}\right\rangle\right) w=v+w,
$$

where $\langle\cdot\rangle$ denotes the statistical expectation value. The homogeneous Boltzmann equation for the distribution function $f=f(w, t)$ corresponding to $(2.2)$ can be easily written in weak form [13]. For all smooth functions $\phi(w)$, the kinetic equation reads

$$
\frac{d}{d t} \int_{\mathbb{R}_{+}} f(w) \phi(w) d w=\left\langle\int_{\mathbb{R}_{+}^{2}}\left(\phi\left(v^{*}\right)-\phi(v)\right) f(v) f(w) d v d w\right\rangle .
$$

Note that, due to (2.3), the total mean wealth is preserved in time

$$
m(t)=\int_{\mathbb{R}_{+}} w f(w, t) d w=m .
$$

The large-time behavior of $f(w, t)[25]$ is largely determined by the convex function

$$
\mathfrak{S}(s):=\gamma^{s}-1+\frac{1}{2}\left\langle\left(1-\gamma+\eta_{1}\right)^{s}+\left(1-\gamma+\eta_{2}\right)^{s}\right\rangle .
$$

Clearly, $\mathfrak{S}(1)=0$ by $(2.3)$. Provided $\mathfrak{S}^{\prime}(0)<0$, the model possesses a unique steady state $f_{\infty}$. If $\mathfrak{S}(s)<0$ for all $s>1$, then $f_{\infty}$ has an exponentially small tail. On the contrary, if there exits a non-trivial root $\bar{s} \in(1, \infty)$ of $\mathfrak{S}$, then $f_{\infty}$ possesses a Pareto tail (2.1) of index $\alpha=\bar{s}$. Moreover, in both cases any solution $f(t)$ converges to $f_{\infty}$ exponentially fast in suitable Fourier and Wasserstein metrics [15].

One further interesting aspect of equation (2.4) is related to the possibility of recovering, in a suitable asymptotic $\left(\gamma \rightarrow 0, \sigma^{2} \rightarrow 0, \sigma^{2} / \gamma \rightarrow \lambda\right)$, a related Fokker-Planck type equation [6,13] for the scaled density $h(v, \tau)=m f(m v, t), \tau=t / \gamma$, which describes the large-time behavior of $f(v, t)$,

$$
\frac{\partial h}{\partial \tau}=\frac{\lambda}{2} \frac{\partial^{2}}{\partial v^{2}}\left(v^{2} h\right)+\frac{\partial}{\partial v}((v-1) h) .
$$

Equation (2.7) admits a unique stationary state of unit mass and unit mean, given by a generalized $\Gamma$-distribution

$$
M_{\lambda}(v)=\frac{(\mu-1)^{\mu}}{\Gamma(\mu)} \frac{\exp \left(-\frac{\mu-1}{v}\right)}{v^{1+\mu}}, \quad \mu=1+\frac{2}{\lambda}>1 .
$$

Note that distribution (2.8) has been first introduced in 1925 by Amoroso [1], exactly in connection with the curve of wealth distribution. This stationary distribution exhibits a Pareto power law tail for large $v$ 's.

We remark that equation (2.7) is essentially the same Fokker-Planck equation derived from a Lotka-Volterra interaction in $[6,30,24]$. Moreover, the asymptotic procedure adopted in [13] leads to the same Fokker-Planck equation even in the case in which the total wealth in trades (2.2) is not preserved.

\subsection{A multi-national kinetic model for the evolution of wealth}

In this section we propose a generalization of the CPT model, where agents from $n$ different countries or social groups of individuals trade with each other. These groups shall be identified with countries or social classes inside a country. We will 
adopt the hypothesis that all agents belonging to one group share a common saving rate parameter. This hypothesis can be further relaxed by assuming that the saving rate is a random quantity, with a statistical mean which is different for different social groups.

This can be seen as the analogue to the physical problem of a mixture of gases, where the molecules of the different gases exchange momentum during collisions [5]. When two agents from the same country with pre-trade wealths $v$ and $w$ interact - a domestic trade event - , then their post-trade wealths $v^{*}$ and $w^{*}$ are supposed to be given by $(2.2)$ with a common saving rate parameter which is characteristic for this country. On the other hand, in case of an international trade, i.e. when two agents of different countries interact, we assume that each agent uses the transaction parameter which is characteristic for his country. Hence, when two agents, one from country $i$ $(i=1,2, \ldots, n)$ with pre-trade wealth $v$ and the other from country $j(j=1,2, \ldots, n)$ with pre-trade wealth $w$ interact, their post-trade wealths $v^{*}$ and $w^{*}$ are given by

$$
\begin{aligned}
v^{*} & =\left(1-\gamma_{i} \gamma\right) v+\gamma_{j} \gamma w+\eta_{i j} v, \\
w^{*} & =\left(1-\gamma_{j} \gamma\right) w+\gamma_{i} \gamma v+\eta_{j i} w .
\end{aligned}
$$

In (2.9), the trade depends on the transaction parameters $\gamma$ and $\gamma_{i}(i=1, \ldots, n)$, while the risks of the market are described by $\eta_{i j}(i, j=1, \ldots, n)$, which are equally distributed random variables with zero mean and variance $\sigma_{i j}^{2}=\lambda_{i j} \gamma$. The different variances for domestic trades in each country and for international trades reflect different risk structures in these trades. For example, investments and trades inside different countries or markets may be subject to different types and quantities of risk, and international trading may face additional risks compared to domestic trades.

The trading rule (2.9) preserves - as in the original CPT model - the total wealth in the statistical mean,

$$
\left\langle v^{*}+w^{*}\right\rangle=\left(1+\left\langle\eta_{i j}\right\rangle\right) v+\left(1+\left\langle\eta_{j i}\right\rangle\right) w=v+w .
$$

In this setting, we are led to study the evolution of the distribution function for each country as a function depending on the wealth $w \in \mathbb{R}_{+}$and time $t \in \mathbb{R}_{+}, f_{i}=$ $f_{i}(w, t)$. In analogy with the classical kinetic theory of mixtures of rarefied gases, the time-evolution of the distributions will obey a system of $n$ Boltzmann-like equations, given by

$$
\frac{\partial}{\partial t} f_{i}(w, t)=\sum_{j=1}^{n} \frac{1}{\tau_{i j}} \mathcal{Q}\left(f_{i}, f_{j}\right)(w), \quad i=1, \ldots, n .
$$

Herein, $\tau_{i j}$ are suitable relaxation times, which depend on the velocity of money circulation [32]. The Boltzmann-like collision operators are derived by standard methods of kinetic theory, considering that the change in time of $f_{i}(w, t)$ due to binary trades depends on a balance between the gain and loss of agents with wealth $w$ [13]. The operator $\mathcal{Q}$ reads

$$
\mathcal{Q}\left(f_{i}, f_{j}\right)(w)=\left\langle\int_{\mathbb{R}_{+}}\left(\frac{1}{J_{i j}} f_{i}\left(v_{*}\right) f_{j}\left(w_{*}\right)-f_{i}(v) f_{j}(w)\right) d v\right\rangle .
$$

In $(2.12),\left(v_{*}, w_{*}\right)$ denote the pre-trade pair that produces the post-trade pair $(v, w)$, following rules like (2.9), while $J_{i j}$ denotes the Jacobian of the transformation of $(v, w)$ 
into $\left(v^{*}, w^{*}\right)$. Finally, $\langle\cdot\rangle$ denotes the operation of mean with respect to the random quantities $\eta_{i j}$. A useful way of writing the collision operator (2.12), that allows to avoid the Jacobian, is the so-called weak form. It corresponds to consider, for all smooth functions $\phi(w)$,

$$
\int_{\mathbb{R}_{+}} \mathcal{Q}\left(f_{i}, f_{j}\right)(w) \phi(w) d w=\left\langle\int_{\mathbb{R}_{+}^{2}}\left(\phi\left(v^{*}\right)-\phi(v)\right) f_{i}(v) f_{j}(w) d v d w\right\rangle .
$$

\section{The continuous trading limit}

In general, it is rather difficult to describe analytically the behavior of the evolution of the wealth densities. As is usual in kinetic theory, it is convenient to study certain asymptotics, which frequently lead to simplified models of Fokker-Planck type. By means of this approach it is easier to identify steady states while retaining important information on the microscopic interaction at a macroscopic level. To this end, we study the continuous trading limit $\left(\gamma \rightarrow 0\right.$ and $\left.\sigma_{i j}^{2} / \gamma=\lambda_{i j}\right)$, following the path laid out in [13].

The weak form of (2.11) is given by

$$
\frac{d}{d t} \int_{\mathbb{R}_{+}} f_{i}(w, t) \phi(w) d w=\int_{\mathbb{R}_{+}} \sum_{j=1}^{n} \frac{1}{\tau_{i j}} \mathcal{Q}\left(f_{i}, f_{j}\right)(w) \phi(w) d w, \quad i=1, \ldots, n,
$$

where the terms on right hand side are given by (2.13). To study the situation for large times, i.e. close to the steady state, we introduce for $\gamma \ll 1$ the transformation

$$
\tau=\gamma t, \quad g_{i}(w, \tau)=f_{i}(w, t), \quad i=1, \ldots, n .
$$

This implies $f_{i, 0}=g_{i, 0}$ and the evolution of the scaled densities $g_{i}(w, \tau)$ follows

$$
\frac{d}{d \tau} \int_{\mathbb{R}_{+}} g_{i}(w, \tau) \phi(w) d w=\frac{1}{\gamma} \int_{\mathbb{R}_{+}} \sum_{j=1}^{n} \frac{1}{\tau_{i j}} \mathcal{Q}\left(f_{i}, f_{j}\right)(w) \phi(w) d w, \quad i=1, \ldots, n .
$$

By the trading rule (2.9), it holds

$$
v^{*}-v=\gamma\left(\gamma_{j} w-\gamma_{i} v\right)+\eta_{i j} v
$$

Using a second order Taylor expansion of $\phi$ around $v$, we obtain

$$
\phi\left(v^{*}\right)-\phi(v)=\phi^{\prime}(v)\left[\gamma\left(\gamma_{j} w-\gamma_{i} v\right)+\eta_{i j} v\right]+\frac{1}{2} \phi^{\prime \prime}(\tilde{v})\left[\gamma\left(\gamma_{j} w-\gamma_{i} v\right)+\eta_{i j} v\right]^{2},
$$

with $\tilde{v}=\theta v^{\prime}+(1-\theta) v$ for some $0 \leq \theta \leq 1$.

Inserting this expansion into the collision operators yields

$$
\begin{aligned}
\frac{d}{d \tau} \int_{\mathbb{R}_{+}} g_{i}(w, \tau) \phi(w) d w & \\
=\frac{1}{\gamma} \sum_{j=1}^{n} \frac{1}{\tau_{i j}}\left\langle\int _ { \mathbb { R } _ { + } ^ { 2 } } \left(\phi^{\prime}(v)\left[\gamma\left(\gamma_{j} w-\gamma_{i} v\right)+\eta_{i j} v\right]\right.\right. & \left.+\frac{1}{2} \phi^{\prime \prime}(v)\left[\gamma\left(\gamma_{j} w-\gamma_{i} v\right)+\eta_{i j} v\right]^{2}\right) \times \\
& \left.\times g_{i}(v, \tau) g_{j}(w, \tau) d v d w\right\rangle+R\left(\gamma, \sigma_{i j}\right),
\end{aligned}
$$


where the remainder $R\left(\gamma, \sigma_{i j}\right)$ converges to zero as $\gamma \rightarrow 0$, provided further hypotheses on the random variables $\eta_{i j}$ and on $\phi$ hold (cf. [13]).

Recalling that $\left\langle\eta_{i j}\right\rangle=0$ and $\left\langle\eta_{i j}^{2}\right\rangle=\lambda_{i j} \gamma$, in the same limit process we obtain

$$
\begin{array}{r}
\lim _{\gamma \rightarrow 0} \frac{1}{\gamma} \sum_{j=1}^{n} \frac{1}{\tau_{i j}}\left\langle\int_{\mathbb{R}_{+}}\left(\phi^{\prime}(v)\left[\gamma\left(\gamma_{j} w-\gamma_{i} v\right)+\eta_{i j} v\right]+\frac{1}{2} \phi^{\prime \prime}(v)\left[\gamma\left(\gamma_{j} w-\gamma_{i} v\right)+\eta_{i j} v\right]^{2}\right) \times\right. \\
\left.\times g_{i}(v, \tau) g_{j}(w, \tau) d v d w\right\rangle \\
=\sum_{j=1}^{n} \frac{1}{\tau_{i j}} \int_{\mathbb{R}_{+}}\left[\phi^{\prime}(v)\left(\gamma_{j} m_{j}-\gamma_{i} v \rho_{j}\right)+\frac{\lambda_{i j}}{2} v^{2} \rho_{j} \phi^{\prime \prime}(v)\right] g_{i}(v, \tau) d v
\end{array}
$$

where

$$
\rho_{j}(\tau)=\int_{\mathbb{R}_{+}} g_{j}(w, \tau) d w, \quad m_{j}(\tau)=\int_{\mathbb{R}_{+}} w g_{j}(w, \tau) d w
$$

denote the mass and the mean wealth of the $j$-th country, respectively. This expression is nothing but the right hand side of the weak form of the system of Fokker-Planck equations

$$
\frac{\partial g_{i}}{\partial \tau}=\sum_{j=1}^{n}\left[\frac{\lambda_{i j}}{2 \tau_{i j}} \frac{\partial^{2}}{\partial v^{2}}\left(v^{2} \rho_{j} g_{i}\right)+\frac{1}{\tau_{i j}} \frac{\partial}{\partial v}\left(\left(\gamma_{i} v \rho_{j}-\gamma_{j} m_{j}\right) g_{i}\right)\right], \quad i=1, \ldots, n .
$$

To formalize the above, let us introduce some notation. Let $\mathcal{M}_{0}$ be the space of all probability measures in $\mathbb{R}_{+}$and

$$
\mathcal{M}_{p}=\left\{\Psi \in \mathcal{M}_{0}: \int_{\mathbb{R}_{+}}|\vartheta|^{p} \Psi(\vartheta) d \vartheta<+\infty, p \geq 0\right\},
$$

the space of all Borel probability measures of finite momentum of order $p$, equipped with the topology of the weak convergence of measures.

Let $\mathcal{F}_{p}\left(\mathbb{R}_{+}\right), p>1$ be the class of all real functions $h$ on $\mathbb{R}_{+}$such that $h(0)=$ $h^{\prime}(0)=0$ and $h^{(m)}(v)$ is Hölder continuous of order $\delta$,

$$
\left\|h^{(m)}\right\|_{\delta}=\sup _{v \neq w} \frac{\left|h^{(m)}(v)-h^{(m)}(w)\right|}{|v-w|^{\delta}}<\infty,
$$

the integer $m$ and the number $0<\delta \leq 1$ are such that $m+\delta=p$, and $h^{(m)}$ denotes the $m$-th derivative of $h$.

Using the same ideas of [13], we obtain in this case the following

Theorem 3.1. Let the probability densities $f_{0, i} \in \mathcal{M}_{p}$, where $p=2+\delta$ for some $\delta>0$, and let the symmetric random variables $\eta_{i j}$ have a density in $\mathcal{M}_{2+\alpha}$ with $\alpha>\delta$. Then, provided $\sigma_{i j}^{2}=\lambda_{i j} \gamma$, as $\gamma \rightarrow 0$, for all $\phi \in \mathcal{F}_{2+\delta}\left(\mathbb{R}_{+}\right)$with $\delta<\alpha$ the weak solutions of the Boltzmann system (2.11) for the scaled densities $g_{i}(w, \tau)=f_{i}(w, t)$ with $\tau=\gamma t$ converge, up to extraction of a subsequence, to probability densities $g_{i}(w, \tau)$. Moreover, these densities are weak solutions of the Fokker-Planck system (3.8).

The (relatively) easy structure of the Fokker-Planck system (3.8) allows to study in some simple case the evolution of the mean wealth in each community, as well as 
the steady state solution. For the sake of simplicity, we will restrict ourselves to the case of two populations, in which $\tau_{i j}=1(i=1,2)$. Then, from system (3.8) it follows that the densities $\rho_{i}(\tau)(i=1,2)$ do not vary with time, while the mean wealths $m_{i}(\tau)$ $(i=1,2)$ satisfy the ordinary differential system

$$
\begin{aligned}
& \frac{d m_{1}}{d \tau}=-\left(\gamma_{1} \rho_{2} m_{1}-\gamma_{2} \rho_{1} m_{2}\right), \\
& \frac{d m_{2}}{d \tau}=+\left(\gamma_{1} \rho_{2} m_{1}-\gamma_{2} \rho_{1} m_{2}\right) .
\end{aligned}
$$

We remark that the evolution of the mean wealths do not depend on the values of the parameters $\lambda_{i j}$. System (3.11) can be solved exactly, to give

$$
\begin{aligned}
& m_{1}(\tau)=a_{1} \frac{\gamma_{2} \rho_{1}}{\gamma_{1} \rho_{2}}-a_{2} e^{-\left(\gamma_{1} \rho_{2}+\gamma_{2} \rho_{1}\right) \tau}, \\
& m_{2}(\tau)=a_{1}+a_{2} e^{-\left(\gamma_{1} \rho_{2}+\gamma_{2} \rho_{1}\right) \tau},
\end{aligned}
$$

with

$$
a_{1}=\left(m_{1,0}+m_{2,0}\right) \frac{\gamma_{1} \rho_{2}}{\gamma_{1} \rho_{2}+\gamma_{2} \rho_{1}}, \quad a_{2}=m_{2,0}-a_{1}
$$

and $m_{1,0}=m_{1}(0), m_{2,0}=m_{2}(0)$. The role of the saving propensity in the evolution of the mean wealth of a population can be clearly recognized as follows. From formulas (3.12) we deduce that, with an exponential rate of convergence, the mean wealths converge towards steady states which are inversely proportional to the $\gamma$ 's (i.e. a bigger $\gamma$ produces a smaller mean wealth),

$$
\begin{aligned}
& m_{1, \infty}=\left(m_{1,0}+m_{2,0}\right) \frac{\gamma_{2} \rho_{1}}{\gamma_{1} \rho_{2}+\gamma_{2} \rho_{1}}, \\
& m_{2, \infty}=\left(m_{1,0}+m_{2,0}\right) \frac{\gamma_{1} \rho_{2}}{\gamma_{1} \rho_{2}+\gamma_{2} \rho_{1}} .
\end{aligned}
$$

This effect can be directly seen also looking at the analytic expressions of the steady states. To this end, let $\rho=\rho_{1}+\rho_{2}$ and $m_{0}=m_{1,0}+m_{2,0}$ denote the initial mass and momentum, and let

$$
\lambda_{i}=\frac{1}{\rho} \sum_{j=1}^{2} \lambda_{i j} \rho_{j}, \quad i=1,2 .
$$

Then, using (3.13), we obtain that the steady states are given by

$$
\begin{aligned}
& g_{1, \infty}(w)=\frac{c_{1}}{\rho} \frac{1}{w^{2+2 \gamma_{1} / \lambda_{1}}} \exp \left\{-\frac{2 \gamma_{1} \gamma_{2} m_{0}}{\lambda_{1}\left(\gamma_{1} \rho_{2}+\gamma_{2} \rho_{1}\right) w}\right\}, \\
& g_{2, \infty}(w)=\frac{c_{2}}{\rho} \frac{1}{w^{2+2 \gamma_{2} / \lambda_{2}}} \exp \left\{-\frac{2 \gamma_{1} \gamma_{2} m_{0}}{\lambda_{2}\left(\gamma_{1} \rho_{2}+\gamma_{2} \rho_{1}\right) w}\right\} .
\end{aligned}
$$

In (3.15) the constants $c_{i}(i=1,2)$ are chosen to have masses $\rho_{1}$ (respectively $\rho_{2}$ ) for the steady states. Note that here the size of the tail of $g_{i, \infty}(w)$ is proportional to $\gamma_{i} / \lambda_{i}$. Hence, in case $\lambda_{i j}=\lambda(i=1,2)$ the smaller the $\gamma$ is, the smaller the number of bounded moments of the steady state is. Taking into account that

$$
\int_{0}^{+\infty} \frac{1}{w^{2+p}} \exp \{-c / w\} d w=\frac{\Gamma(p+1)}{c^{p+1}},
$$


where $\Gamma(\cdot)$ is Euler's gamma function, one can easily recover the value of the constants $c_{i}$,

$$
c_{i}=\frac{\rho_{i}}{\Gamma\left(1+2 \gamma_{i} / \lambda_{i}\right)}\left(\frac{2 \gamma_{1} \gamma_{2} m_{0}}{\lambda_{i}\left(\gamma_{1} \rho_{2}+\gamma_{2} \rho_{1}\right)}\right)^{1+2 \gamma_{i} / \lambda_{i}}, \quad i=1,2
$$

Taking the sum of the densities in (3.15) gives the expression of the total density

$$
g_{\infty}(w)=\frac{1}{\rho}\left(\frac{c_{1}}{w^{2+2 \gamma_{1} / \lambda_{1}}} e^{-d_{1} / w}+\frac{c_{2}}{w^{2+2 \gamma_{2} / \lambda_{2}}} e^{-d_{2} / w}\right),
$$

where we defined

$$
d_{i}=\frac{2 \gamma_{1} \gamma_{2} m_{0}}{\lambda_{i}\left(\gamma_{1} \rho_{2}+\gamma_{2} \rho_{1}\right)}, \quad i=1,2
$$

In the simplest case in which $\lambda_{i}=\lambda$, the two steady states have a common exponential factor, and

$$
g_{\infty}(w)=\frac{1}{\rho}\left(\frac{c_{1}}{w^{2+2 \gamma_{1} / \lambda}}+\frac{c_{2}}{w^{2+2 \gamma_{2} / \lambda}}\right) \exp \left\{-\frac{2 \gamma_{1} \gamma_{2} m_{0}}{\lambda\left(\gamma_{1} \rho_{2}+\gamma_{2} \rho_{1}\right) w}\right\}
$$

The analysis of the steady density (3.17) reveals that, depending of the values of the various parameters involved, one can have the formation of a bimodal distribution. In the simplified situation in which $\lambda_{i}=\lambda$, one can recover a more precise behavior of the steady state. In this case in fact, using (3.16), the steady state (3.19) results to be a (nonnegative) function of the form

$$
\psi(w)=\left(\frac{a^{2} c^{p+1}}{w^{p+2} \Gamma(p+1)}+\frac{b^{2} c^{q+1}}{w^{q+2} \Gamma(q+1)}\right) \exp \{-c / w\},
$$

where $a^{2}+b^{2}=1$. With the substitution $x=w / c$, we obtain for (3.20) the simpler form

$$
\Psi(x)=\frac{1}{c}\left(\frac{a^{2} x^{-(p+2)}}{\Gamma(p+1)}+\frac{b^{2} x^{-(q+2)}}{\Gamma(q+1)}\right) \exp \{-1 / x\} .
$$

The extremals of (3.21) are located in the points that are solutions of the equation

$$
\left(-\frac{a^{2}(p+2) x^{-(p+3)}}{\Gamma(p+1)}-\frac{b^{2}(q+2) x^{-(q+3)}}{\Gamma(q+1)}+\frac{a^{2} x^{-(p+4)}}{\Gamma(p+1)}+\frac{b^{2} x^{-(q+4)}}{\Gamma(q+1)}\right) e^{-1 / x}=0 .
$$

Clearly, $x=0$ is an extremal (a minimum). If $x>0$, and $p<q$, the remaining extrema are located in the points solutions of the equation

$$
\Phi(x)=-\frac{a^{2}(p+2) x^{q-p+1}}{\Gamma(p+1)}-\frac{b^{2}(q+2) x}{\Gamma(q+1)}+\frac{a^{2} x^{q-p}}{\Gamma(p+1)}+\frac{b^{2}}{\Gamma(q+1)}=0 .
$$

On the other hand, since $\Phi(0)>0$, while $\Phi(+\infty)=-\infty$, the curve $y=\Phi(w)$ touches the axis $y=0$ either in a single point, or in three points. In this last case, we have two maxima and one minimum outside $w=0$, and consequently a bimodal distribution. 


\section{A model for distributed trading propensity}

The Fokker-Planck system (3.8) can be easily generalized to the case in which the trading rate is randomly distributed on the interval $(0,1)$, with distribution $\Gamma(s)$, where

$$
\Gamma(s)=\int_{\mathbb{R}_{+}} g_{0}(v, s) d v, \quad 0<s<1
$$

is the $s$-marginal of the initial density of wealth. In this case, the unknown densities $g_{i}(v, \tau)(i=1, \ldots, n)$ are substituted by $g(v, s, \tau)$, while $\sum_{j=1}^{n} g_{i}$ move to

$$
\langle g(v, s)\rangle_{s}=\int_{0}^{1} g(v, s) d s
$$

Note that here and below we will denote

$$
\langle\Phi(s)\rangle_{s}=\int_{0}^{1} \Phi(s) d s .
$$

If we assume $\lambda_{i j}=\lambda, \tau_{i j}=1(i=1, \ldots, n)$ in (3.8), the corresponding Fokker-Planck equation with a continuous varying trading rate reads

$$
\frac{\partial g(v, s)}{\partial \tau}=\frac{\lambda}{2} \frac{\partial^{2}}{\partial v^{2}}\left(v^{2}\langle\rho(s, \tau)\rangle_{s} g(v, s)\right)+\frac{\partial}{\partial v}\left(\left(s v\langle\rho(s, \tau)\rangle_{s}-\langle s m(s, \tau)\rangle_{s}\right) g(v, s)\right) .
$$

Taking into account that the total mass is preserved, $\langle\rho(s)\rangle_{s}=1$, while the distribution

$$
\Gamma(s, \tau)=\int_{\mathbb{R}_{+}} g(v, s, \tau) d v
$$

does not depend on time, i.e. $\Gamma(s, \tau)=\Gamma(s)$, equation (4.1) simplifies to

$$
\frac{\partial g(v, s)}{\partial \tau}=\frac{\lambda}{2} \frac{\partial^{2}}{\partial v^{2}}\left(v^{2} g(v, s)\right)+\frac{\partial}{\partial v}\left(\left(s v-\langle s m(s)\rangle_{s}\right) g(v, s)\right) .
$$

A related Boltzmann type model, based on the binary trades with distributed saving propensities introduced by Chakrabarti, Chatterjee and Manna [9], has been recently analytically investigated in [26]. In particular, necessary conditions on the distribution $\Gamma(s)$ to guarantee convergence towards a steady state have been discussed, together with the steady state stability. It could be certainly interesting to investigate if a similar analysis could be done for the Fokker-Planck model (4.2), to verify in particular if a bimodal distribution can result for particular choices of the distribution $\Gamma$. This would give a theoretical basis to the numerical experiments of Gupta [21], who remarked that one still gets a two-peak distribution even when the two saving propensities $\gamma_{1}$ and $\gamma_{2}$ are drawn from narrow distributions centered around two widely separated values (one large and one small).

\section{Numerical results}

To illustrate the relaxation behavior and to study the influence of the different model parameters, we have performed a series of kinetic Monte Carlo simulations for the Boltzmann model presented in the previous section. We will focus on the situation 
of two countries, i.e. $n=2$. It will be straightforward, however, to extend the following to the general situation of an arbitrary number of countries. Hence, let us consider

$$
\begin{aligned}
\frac{\partial}{\partial t} f_{1}(w, t) & =\frac{1}{\tau_{11}} \mathcal{Q}\left(f_{1}, f_{1}\right)(w)+\frac{1}{\tau_{12}} \mathcal{Q}\left(f_{1}, f_{2}\right)(w), \\
\frac{\partial}{\partial t} f_{2}(w, t) & =\frac{1}{\tau_{22}} \mathcal{Q}\left(f_{2}, f_{2}\right)(w)+\frac{1}{\tau_{21}} \mathcal{Q}\left(f_{2}, f_{1}\right)(w) .
\end{aligned}
$$

Herein, $\mathcal{Q}\left(f_{1}, f_{1}\right)$ and $\mathcal{Q}\left(f_{2}, f_{2}\right)$ represent the collision operators which describe the change of density due to binary domestic trades, while $\mathcal{Q}\left(f_{1}, f_{2}\right), \mathcal{Q}\left(f_{2}, f_{1}\right)$ are the collision operators which describe the change of density due to binary international trades.

Generally, in this kind of simulations, known as direct simulation Monte Carlo (DSMC) or Bird's scheme, pairs of agents are randomly and non-exclusively selected for binary collisions, and exchange wealth according to the trading rule under consideration. To extend this procedure to the present situation, we pursue the following approach. Let us denote by $N_{i}(i=1,2)$ the number of traders of the two countries we consider in our simulation. Assume without loss of generality, that $N_{1} \geq N_{2}$. One time step in our simulation corresponds to $N_{1}$ interactions. Since we have to perform trade events for both groups, each of these interactions has two stages, which are described in the following.

In the first stage, select randomly an agent from group 1. Then select randomly a trading partner from the whole population, where the probabilities for each agent to be selected depend on $\tau_{11}, \tau_{12}$. For example, if the trading frequency in group 1 for domestic trades is twice as high as for international trades, the probability to select a trade partner from group 1 has to be twice the probability to select a trade partner from group 2. Once the trade partner is selected, the trade takes place and wealth is exchanged according to the trading rule (2.9).

In the second stage, we need to perform trades for group 2. Since $N_{2} \leq N_{1}$, we only perform a trade for group 2 in every $k$-th interaction, where $k=\left\lceil N_{1} / N_{2}\right\rceil$. If a trade is carried out, it is done similarly as for group 1: select randomly an agent from group 2 and a partner from the whole population, where the probabilities for an agent to be selected as a partner depend on $\tau_{21}, \tau_{22}$. Then, carry out the trade according to the trading rule $(2.9)$.

In all our experiments, every agent possesses unit wealth initially. The relaxation in the CPT model occurs exponentially fast [15]. Hence, to compute a good approximation of the steady state it suffices to carry out the simulation for about $10^{4}$ time steps, and then average the wealth distribution over another 1000 time steps. In every experiment, we average over $M=100$ such simulation runs.

We consider two groups with $N_{1}=N_{2}=5000$ agents. We investigate the relaxation behavior when the random variables $\eta_{i j}, i, j \in\{1,2\}$, attain values $\pm \mu$ with probability $1 / 2$ each. We set the coefficient $\gamma=1$. Let $\mu=0.15$ and $\tau_{i j}=1$ for $i, j \in\{1,2\}$. If we choose $\gamma_{1}=\gamma_{2}=0.125$ and $\gamma_{1}=\gamma_{2}=0.01$, respectively, the system reduces to the standard CPT model. The probability density for both cases is plotted in Figure 5.1. The cumulative distribution functions show a Pareto tail; see Figure 5.2. The Pareto index $\alpha$ of the tail is determined by the non-trivial root of (2.6) - strictly speaking this holds for the limit $N_{1,2} \rightarrow \infty-$, which is given by 28.068 and 1.875 , respectively. These tail indices are indicated in Figure 5.2 by a thick line.

Now, we choose $\gamma_{1}=0.125$ and $\gamma_{2}=0.01$ and keep $\mu=0.15$ and $\tau_{i j}=1$ for $i, j \in$ $\{1,2\}$. The probability density for the whole population is plotted in Figure 5.3 (left 

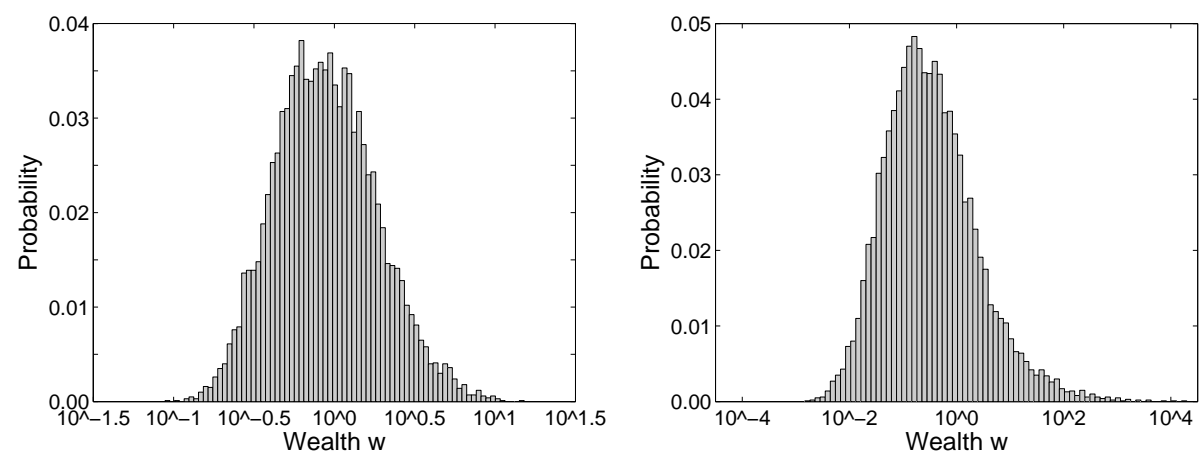

FIG. 5.1. Histogram of steady state distribution for $\gamma_{1}=\gamma_{2}=0.125$ (left) and for $\gamma_{1}=\gamma_{2}=0.01$ (right).
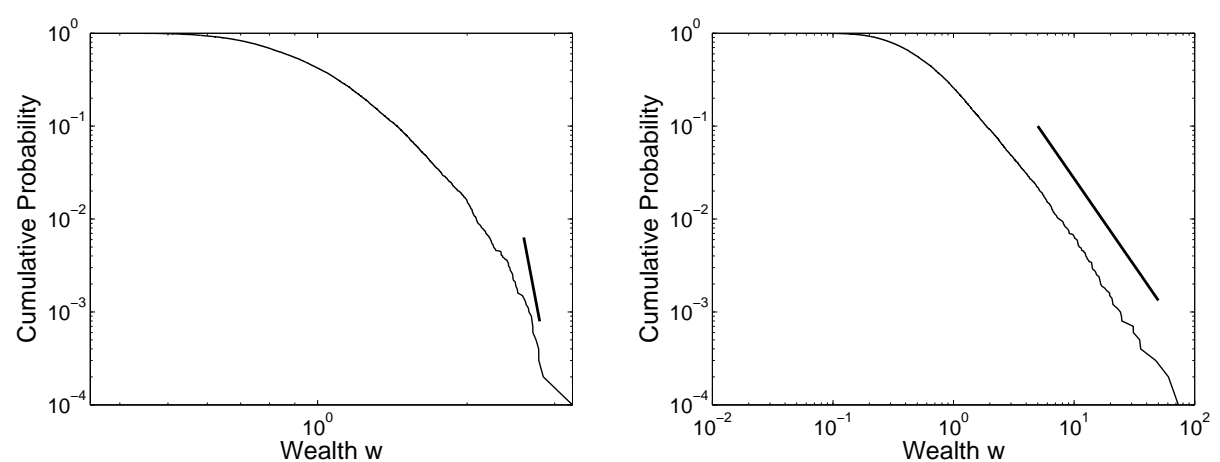

FIG. 5.2. Cumulative wealth distribution for $\gamma_{1}=\gamma_{2}=0.125$ (left) and for $\gamma_{1}=\gamma_{2}=0.01$ (right).

plot). It shows a bimodal shape. Comparative simulations show that the distance of the two peaks in the distribution decreases with decreasing difference between $\gamma_{1}$ and $\gamma_{2}$. This observation is consistent with the result (3.13) for the mean wealth of the steady state in the Fokker-Planck limit. Such bimodal distributions (and a polymodal distribution, in general) are also reported with real data for the income distributions in Argentina [20, 21]. This distribution features transport of wealth from one group to the other, which makes it different from the probability distribution for the union of two groups with the same parameters which do not interact, see Figure 5.3 (right plot).

The associated cumulative distribution functions are shown in Figure 5.4. Both curves are dominated by the tail behavior of the second group with smaller $\gamma$ and show a Pareto tail of the respective index.

The distribution and the cumulative distribution functions for the two groups are shown in Figure 5.5 and 5.6. For comparison we plot the same Pareto tail index lines as in Figure 5.2. Apart from a 'kink' in the tail for the first group which represents a finite-size effect, the cumulative distributions show Pareto tails with the respective index. This is in nice agreement with the theoretical results of Section 3 for the steady state in the Fokker-Planck limit. 

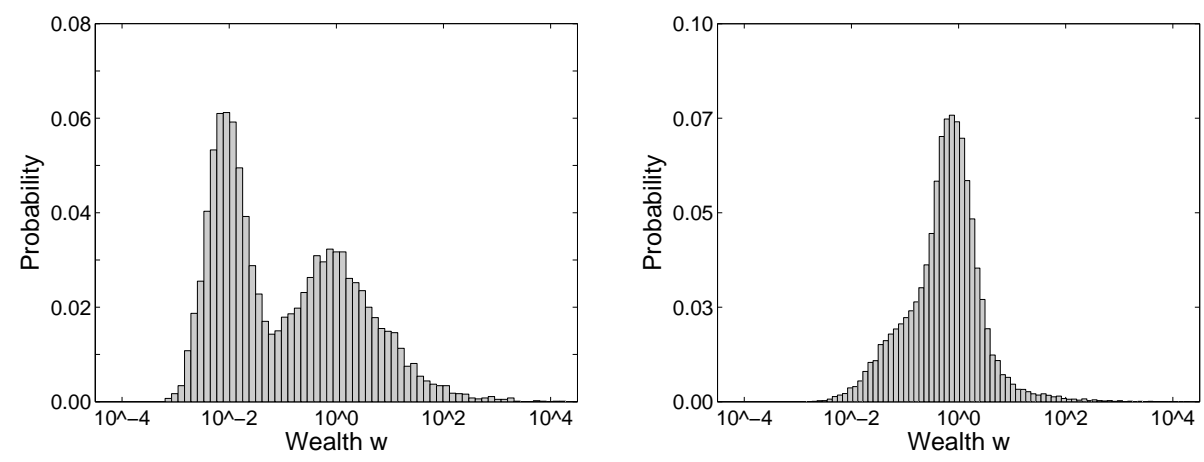

FIG. 5.3. Histogram of steady state distribution for $\gamma_{1}=0.125$ and $\gamma_{2}=0.01$ (left) in comparison with the histogram for the union two disjoint populations with the same parameters (right).
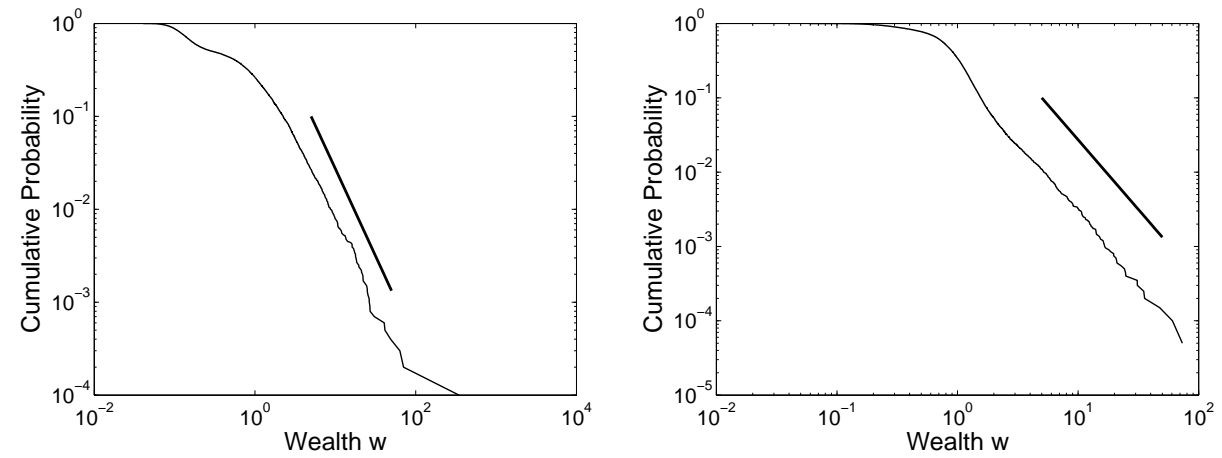

FIG. 5.4. Cumulative wealth distribution for $\gamma_{1}=0.125$ and $\gamma_{2}=0.01$ (left) in comparison with the cumulative wealth distribution for the union two disjoint populations with the same parameters (right).
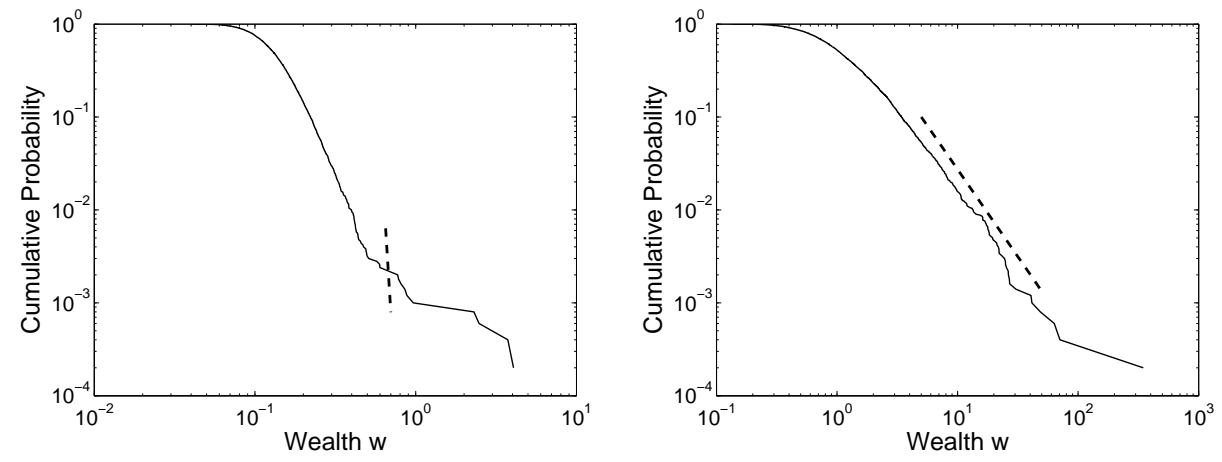

FiG. 5.5. Cumulative wealth distribution for group 1 with $\gamma_{1}=0.125$ (left) and group 2 with $\gamma_{2}=0.01$ (right). 

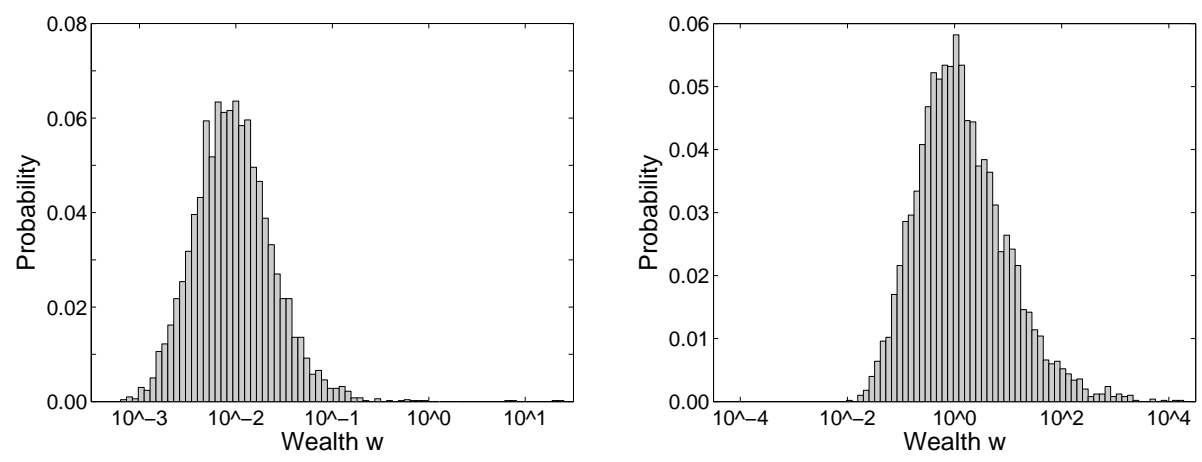

FIG. 5.6. Wealth distribution for group 1 with $\gamma_{1}=0.125$ (left) and group 2 with $\gamma_{2}=0.01$ (right).
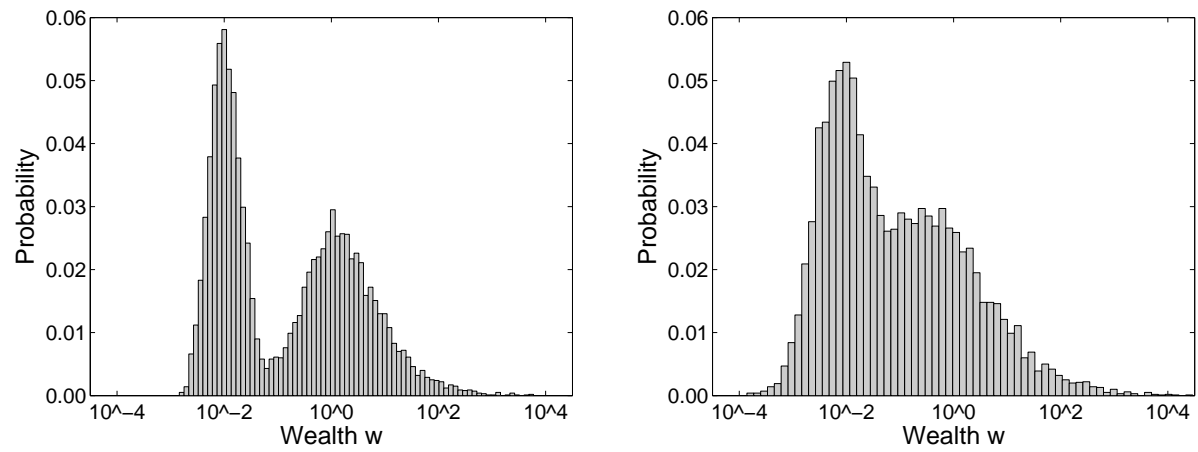

FIG. 5.7. Influence of $\eta_{i j}$ : Wealth distribution with $\gamma_{1}=0.125, \gamma_{2}=0.01$ with $\eta_{12}=\eta_{21}=0.075$ (left) and $\eta_{12}=\eta_{21}=0.225$ (right) and $\eta_{11}=\eta_{22}=0.15$ in both cases.

To illustrate the influence of the risk parameter $\eta_{i j}$, we perform simulations with increased and decreased risk for international trades, i.e. we choose $\eta_{12}=\eta_{21}=0.075$ and $\eta_{12}=\eta_{21}=0.225$, respectively, while we keep the other parameters unchanged. The wealth distributions are shown in Figure 5.7. For $\eta_{12}=\eta_{21}=0.075$, the bimodal profile is more pronounced, while the additional diffusion in the case $\eta_{12}=\eta_{21}=0.225$ tends to blur the bimodal shape.

6. Conclusions We introduced and discussed a nonlinear kinetic model for a simple market economy which is built of a number of countries or social groups. The evolution of wealth is described by a system of Boltzmann-like equations in which collisions describe binary exchanges of wealth and speculative trading, and different social groups are characterized by a personal saving rate. Following the ideas of [13] we showed that at suitably large times, in presence of a large number of trades in which agents exchange small amounts of wealth, the nonlinear system of Boltzmann-type equations is well-approximated by a system of linear Fokker-Planck type equations, which admits a stationary steady state with Pareto tails. Convergence towards a similar steady state is shown numerically for the solution of the kinetic model. In case of two groups of agents, both the numerical simulation on the kinetic model, and the analytical study of the underlying Fokker-Planck system reveal formation 
of a bimodal distribution, giving a theoretical framework to previous works on the subject $[21,24]$, where the appearance of bimodal distributions has been observed only numerically.

Acknowledgement. The authors acknowledge support from the Italian MIUR, project "Kinetic and hydrodynamic equations of complex collisional systems". Bertram Düring is partly supported by the Deutsche Forschungsgemeinschaft, grant JU 359/6 (Forschergruppe 518), and by the ESF Project GLOBAL. Bertram Düring thanks the Department of Mathematics of the University of Pavia, where a part of this research has been carried out, for the kind hospitality.

\section{REFERENCES}

[1] L. Amoroso, Ricerche intorno alla curva dei redditi, Ann. Mat. Pura Appl. Ser. 4, 21, 123-159, 1925.

[2] M. Bisi, J.A. Carrillo, and G. Toscani, Decay rates in probability metrics towards homogeneous cooling states for the inelastic Maxwell model, J. Statist. Phys., 124, 625-653, 2006.

[3] A.V. Bobylev and C. Cercignani, Self-similar asymptotics for the Boltzmann equation with inelastic and elastic interactions, J. Statist. Phys. 110, 333-375, 2003.

[4] A.V. Bobylev, C. Cercignani, and G. Toscani, Proof of an asymptotic property of self-similar solutions of the Boltzmann equation for granular materials, J. Statist. Phys. 111, 403-417, 2003.

[5] A.V. Bobylev and I.M. Gamba, Boltzmann equations for mixtures of Maxwell gases: exact solutions and power like tails, J. Stat. Phys., 124, 497-516, 2006.

[6] J.P. Bouchaud and M. Mézard, Wealth condensation in a simple model of economy, Physica A, 282, 536-545, 2000.

[7] A. Chakraborti, Distributions of money in models of market economy, Int. J. Modern Phys. C, 13, 1315-1321, 2002.

[8] A. Chakraborti and B.K. Chakrabarti, Statistical Mechanics of Money: Effects of Saving Propensity, Eur. Phys. J. B, 17, 167-170, 2000.

[9] A. Chatterjee, B.K. Chakrabarti, and S.S. Manna, Pareto Law in a Kinetic Model of Market with Random Saving Propensity, Physica A, 335, 155-163, 2004.

[10] A. Chatterjee, S. Yarlagadda, and B.K. Chakrabarti, Econophysics of Wealth Distributions, New Economic Window Series, Springer, 2005.

[11] A. Chatterjee, B.K. Chakrabarti, and R.B. Stinchcombe, Master equation for a kinetic model of trading market and its analytic solution, Phys. Rev. E, 72, 026126, 2005.

[12] R. Coelho, P. Richmond, J. Barry, and S. Hutzler, Double power law in income and wealth distributions, Physica A, 387, 3847-3851, 2008.

[13] S. Cordier, L. Pareschi, and G. Toscani, On a kinetic model for a simple market economy, J. Stat. Phys., 120, 253-277, 2005.

[14] A. Drăgulescu and V.M. Yakovenko, Statistical mechanics of money, Eur. Phys. Jour. B, 17, 723-729, 2000.

[15] B. Düring, D. Matthes, and G. Toscani, Exponential and algebraic relaxation in kinetic models for wealth distribution, in: "WASCOM 2007" - Proceedings of the 14th Conference on Waves and Stability in Continuous Media, N. Manganaro et al. (eds.), World Sci. Publ., Hackensack, NJ, 2008.

[16] B. Düring, D. Matthes, and G. Toscani, Kinetic equations modelling wealth redistribution: a comparison of approaches, preprint, 2008.

[17] B. Düring and G. Toscani, Hydrodynamics from kinetic models of conservative economies, Physica A, 384(2), 493-506, 2007.

[18] M.H. Ernst and R. Brit, High energy tails for inelastic Maxwell models, Europhys. Lett., 58, 182-187, 2002.

[19] M.H. Ernst and R. Brito, Scaling solutions of inelastic Boltzmann equation with over-populated high energy tails, J. Statist. Phys., 109, 407-432, 2002.

[20] J.C. Ferrero, The monomodal, polymodal, equilibrium and nonequilibrium distribution of money, in: Econophysics of Wealth Distributions, A. Chatterjee, S. Yarlagadda, and B.K. Chakrabarti (eds.), Springer (Italy), 2005.

[21] K. Gupta, Money exchange model and a general outlook, Physica A, 359, 634-640, 2006.

[22] B. Hayes, Follow the money, American Scientist, 90(5), 400-405, 2002. 
[23] S. Ispolatov, P.L. Krapivsky, and S. Redner, Wealth distributions in asset exchange models, Eur. Phys. Jour. B, 2, 267-276, 1998.

[24] O. Malcai, O. Biham, P. Richmond, and S. Solomon, Theoretical analysis and simulations of the generalized Lotka-Volterra model, Phys. Rev. E, 66, 031102, 2002.

[25] D. Matthes and G. Toscani, On steady distributions of kinetic models of conservative economies, J. Stat. Phys., 130, 1087-1117, 2008.

[26] D. Matthes and G. Toscani, Analysis of a model for wealth redistribution, Kinetic and related Models, 1, 1-22, 2008.

[27] L. Pareschi and G. Toscani, Self-similarity and power-like tails in nonconservative kinetic models, J. Stat. Phys. 124(2-4), 747-779, 2006.

[28] V. Pareto, Cours d'Economie Politique, Lausanne and Paris, 1897.

[29] F. Slanina, Inelastically scattering particles and wealth distribution in an open economy, Phys. Rev. E, 69, 046102, 2004.

[30] S. Solomon, Stochastic Lotka-Volterra systems of competing auto-catalytic agents lead generically to truncated Pareto power wealth distribution, truncated Levy distribution of market returns, clustered volatility, booms and crashes, in: Decision technologies for Computational Finance, A-P.N. Refenes, A.N. Burgess, and J.E. Moody (eds.), Kluwer Academic Publ., 1998.

[31] S. Solomon and P. Richmond, Stable power laws in variable economies; Lotka-Volterra implies Pareto-Zipf, Eur. Phys. J. B, 27, 257-262, 2002.

[32] Y. Wang, N. Ding, and L. Zhang, The circulation of money and holding time distribution, Physica A, 324(3-4), 665-677, 2003. 